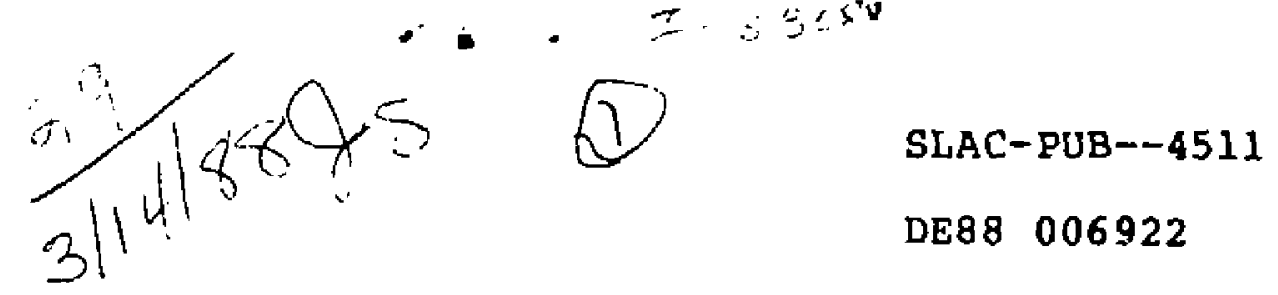

\title{
MEASUREMENT OF THE B HADRON \\ LIFETIME FROM MARK II AT PEP*
}

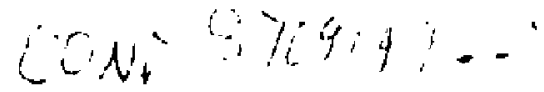

RENE A. ONG

Stanford Linear Accelerator Center

Stanford Universily, Stanford, California 94905

(Representing the Mark In Collaboration ${ }^{191}$ )

\begin{abstract}
Using dnta taken by the Mark Il detector at PEP, the decayo of $B$ hadronis are toged by identifying leptons at high transverae momentum. By mears of a precision inner drift chamber, the impact purameters of these leptons are measured with reapeet to the B production point. From this impact parameter distribution, the $B$ hadron lifetime is found to be $0.98 \pm 0.12 \pm 0.13$ po. This measurement can be used to place constraints models of quarh mixing.
\end{abstract}

Invited talk presented at the International Symposium on The Production and Decay of Heavy Flavord

Stanjord, California, September $1-5,1987$

- This work -4u enported in part by the Depertment of Energy, contrucu DE-AC03-76SFDOS 15 (5LAC), DE-AC03-76SF00098 (LBL), and DE ACO2-76ERO3039 (Harvard). 


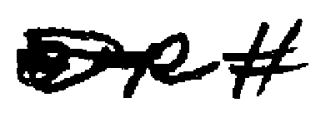

\section{$04 / 2-x^{-}$}

\section{Introduction}

The B hadron lifetime is important berause it is a measure of the otrength of thr weak trangitions between quarkg. Within the aix quark mixing scheme proprited hy Kobayashi and Maskawa, ${ }^{\text {') }}$ the $B$ lifetime measures the matrix elements $\left|V_{a b}\right|$ and $\left|V_{c b}\right|$ dencribing $(b \rightarrow u)$ and $(b \rightarrow c)$ transitions, respectively. There cre numerous calculatic is of the expected $B$ decay rate within the $S$ tandard Model. These calculations have used the free quark model for $\mathrm{B}$ hadron decay ${ }^{|2|}$ and modela deacribing transitions between exclusive hadronic states. ${ }^{\text {ol }}$

There are number of expetimentat complications that arise in the disterminstion of the B lifetime. At PEP/PETRA energies, bib events canstitute only $\sim 10 \%$ of the total hadronic event sumple. The low fraction of $b \bar{b}$ events, the relatively high multiplicity of $B$ decays, and the propensity of $B$ hadront to produce neutral purticles have made full reconatruction of exclunive decaya difficult. It is therefore valuable to enrich the hadronic axmple in $b \bar{b}$ events in order to maximize senaitivity to the $\mathbf{B}$ lifetime, in the Mark Il analysis presented here, this enrichment is done by takging high transverse momentum leptons from $\mathrm{a}$ decay. The impect parameters of there tepton tracks measured relative to the $B$ production point are then used to determine the $B$ lifetime.

Previoun measurements of the $B$ lifetime have been made by numerous experiments at PEP and PETRA. The most recent reaults are given in Ref. 5. These mesuremento find B lifelime values in the 1.0 to 1.5 pa range and employ diverse techniques to extract the lifetime from a hadronic event sample.

In this talk, the final B lifetime measurement from the Mark it detector it PEP is reported. ${ }^{\text {Io }}$ The detector is briefly described, along with studies mide to understand the experimental resolution. An analyais of inclusive leptons produced at $29 \mathrm{GeV}$ is discuased. The method of impact par urneter meanurement is presented and the lifetime is extracted by means of a maximum likelihnod fit to the lepton impact parameter distribution. The gystematic errors associated with the lifetime determination are summarized at the end of the talk. 


\section{Mark II Detector}

The Mark 11 detector has been described in detait elsewhere. "I Electrons are identified by requiring consistency between the momentum of a track as measured in the central drift chamber and the corresponding track energy measured in a Pb-Isiquid Argon calorimeter. ${ }^{|0|}$ Muons are identified in a system of haritrir absorber and proportional tubes.

Of primary ifoportance for this analysis is a high precision inner drift chamber or Vertex Chamber (VC). ${ }^{[0]}$ The VC has seven layers of axial wires strung between 11 and $32 \mathrm{~cm}$ from the interaction point. From Bhabha geparation digtances, the impact parameter resolution of the VC for high momentum tracks is found to be $85 \mu \mathrm{m}$. The resolution function for tracks in hadronic even " (oimitar to those used in the lifetime analyuis) is determined from the impact parameter/erfor distribution of trachs hoving a small fraction of their trassverse momentum in the $x y$ plane." Thia resolution function is found to have a larger width and more tail than the function describing Bhabhe tracks.

\section{Inclusive Lepton Analysis}

The Mark II detector at PEP accumulated an integrated luminosity of 204 $\mathrm{pb}^{-1}$ at $\mathrm{E}_{\mathrm{ctm}}=29 \mathrm{GeV}$. Using this data, 2631 electron and 1230 muon candidales with momentum greater than $2 \mathrm{GeV} / c$ are isolated. The lepton $\left(p, p_{i}\right)$ spectrum is fit in terms of its various contributions from charm decay, bottom decaya, and background sources. From the fit, the semi-leptonts charm and bottom hadron branching ratios are determined, along with the average badron energy $(\langle z\rangle$ of fragmentation). ${ }^{\dagger}$ Since $B$ decays are not fully reconstructed, knowledge of the fragmentation $\langle z\rangle$ is crucial in order to convert an estimator such as impact parameter to lifetime. The results of the inclusive lepton analysis are

\footnotetext{
- Thio criterion aelects a asmple of tracks with little lifetime information in the $x y$ plane, which is Lise VC measurement plane

$f$ " only $\left\langle z_{b}\right\rangle$ is determined froni the frt; the value of $\left\langle z_{r}\right\rangle \quad 9.68$, deteranined from exclusive tham decayn, is lixed
} 
given in Table 1. They are in good agreement with those obtained by other experiments. ${ }^{|\operatorname{lo}|}$

Table 1. Results from the MARK II inclusive lepton aralysis.

\begin{tabular}{|c|c|c|}
\hline Quantity & Electron & Muon \\
\hline $\operatorname{BR}(c \rightarrow l)$ & $9.6 \pm 0.7 \pm 1.5(\%)$ & $7.8 \pm 0.9 \pm 1.2(\%)$ \\
$\operatorname{BR}(b \rightarrow l)$ & $11.2 \pm 0.9 \pm 1.1(\%)$ & $11.8 \pm 1.2 \pm 1.0(\%)$ \\
$\left\langle z_{b}\right\rangle$ & $0.85 \pm 0.03 \pm 0.05$ & $0.82 \pm 0.04 \pm 0.03$ \\
\hline
\end{tabular}

The lepton $p_{1}$ spectra are illustrated in Figure 1. From this figure, it can be seen that the majority of leptons at high transverse momentum come from $B$ decays. $A$ B enhanced sample is defined by requiring leptons to have $p_{t}$ greater than $1 \mathrm{GeV} / \mathrm{c}$. In this sample, $65 \pm 5 \%$ of the leptons come from primary or secondary $B$ hadron decays; the remaining leptons come approximately equally from $\mathbf{C}$ hadron decays or background.

\section{Lifetime Determination}

We have seen that tagging leptons at high $p_{t}$ serves to enrich a hadronic event sample in $B$ hadron decays. These leptons are used to extimate the $B$ lifetime by the calculation of a signed impact parameter. The impact parameter is measured in the $x y$ plane with respect to the presumed $B$ production point. The thruat axis of the event serves to estimate the $B$ flight direction and to determine the impact parameter sign. The impact parameter is signed positive if the intemection point of lepton trajectory and tine assumed B trajectory corresponds to a positive decay length and is signed negative otherwige.

The beam position (determined from Bhabha tracks over many events) can be used as an estimate for the B production point. We improve upon this estimate by the use of a vertex method. Each event is divided into two hernisplieses 'n : 
plane perpendicular to the thrust axis. $A$ vertex is made of the quality tracks in each jet. Using this vertex, the thrust direction, and the beam position as inputs to the decay length method, ${ }^{[1]}$ an estimate is made of the B production point. A variety of checks in the data and Monte Carlo show that this precedure produces an unbiased estimate of the $B$ production point. The lepton impact parameter is mexsured with respect to the production point. $A$ factor of two gain in impact parameter precision is oblained by using the estimated production point over the beam centroid. This gain results from the reduction in the error due to the borizontal beam size.

The impact parameter distribution for the 617 leptons in the $B$ enhanced sample is shown in Figure 2. With the aid of the Monte Carlo, a maximum likelihood fit to this distribution yields:

$$
r_{1}=0.98 \pm 0.12 \pm 0.13 \text { psec }
$$

where the firot error is statistical and the second is systematic. In the fit, the resolution function discussed in Section 2 is used. This funciion accounts for noin-Gaussinn tails in the reaolution.

A summary of the syotematic errors affecting the lifetime mensurement are presented in Table 2. The overal systematic error is largely determined by uncertaintie in the resolution, fragmentation, and lepton fractions.

Table 2. Systemutic errors fot the MARK II lifetime mesaurement.

\begin{tabular}{|c|c|}
\hline Quantity & Error on Lifetime \\
\hline Rewolution uncertainty & $\pm 0.07 \mathrm{psec}$ \\
B fragmentation, $<z>=0.83 \pm 0.07$ & $\pm 0.05 \mathrm{psec}$ \\
Lepton (B) fractione & $\pm 0.08 \mathrm{psec}$ \\
Other & $\pm 0.04 \mathrm{psec}$ \\
\hline Total & $\pm 0.13 \mathrm{psec}$ \\
\hline
\end{tabular}




\section{Conclusions}

The B lifetime measurement presented in this talk is consiatent with those from other experiments. Using the calculation of Ref. [3], the sesults of this measurement imply a value of $\left|V_{c t}\right|=0.0 .54 \pm 0.003 \pm 0.004 .^{\circ}$ The amallness of $\left|V_{c b}\right|$ in relation to the Cabibbo angle indicates that the mixing between the third generation of quarks and the lighter generations is much weaker than that between the first two generations.

\section{Acknowledgement.}

1 have benefited from uneful diacussions with John Jaros and Harry Nelson.

\section{References}

1. The members of the Mark II Collaboration are: G.S. Abrams, D. Amidei, A.R. Baden, T. Barklow, A.M. Boyarski, J. Boyer, P.R. Burchat, D.L. Burke, F. Butler, J.M. Dorfan, G.J. Foldman, G. Gidal, L. Gladney, M.S. Gold, G. Goldhaber, L. Golding, J, Haggerty, G. Hasaon, K. Hayes, D. Herrup, R.J. Hollebeek, W.R. Innes, J.A. Jeroe, I. Juricic, J.A. Kadyk, D. Karlen, S.R. Klein, A.J. Lentford, R.R. Larsen, B.W. LeCleire, M. Levi, ri.S. Lockyer, V. Lüth, C. Matteuzzi, M.E. Nelson, R.A. Ong, M.L. Perl, A. Peterten, B. Richter, K. Riles, P.C. Rowson, T. Schasd, H. Schellman, W.B. Schmidke, P.D. Sheldon, G.H. Trilling, C. de is Vaissiere, D.R. Wood, and J.M. Yelton

2. M. Kobayaghi and T. Maskawa, Prog. Theor. Phys. 49, 652 (1973).

3. See, for example: G. Altarelli et al., Nucl. Phys. B208, 365 (1982).

- The value of $\left|V_{c b}\right|$ in obinined asuming $\left|V_{a b}\right| /\left|V_{c B}\right|=0.15$, conasistent wh the reaulta presented by CLEO and ARGUS at this Symposium. 
4. M. Wirbel, B. Stech, and M. Bauer, 2. Phys, C20, 637 (1985);

B. Grinstein, M.B. Wise, and N. Iggur, Phys. Rev. Lett. 56, 298 (1986).

5. D.E. Klem et al., SLAC-Pub-4025, submitted to Phys. Rev, D. W.W. Ash te al., Phys. Rev. Lett. 58, 640 (1987);

J.-M. Brom et al., Phys. Lett. B195, 301 (1987);

S.L. Wu, Procedings of the 1987 International Symposium on Lepton and Photon Interactions at High Energies, (Hamburg, 1987).

6. R.A. Ong, Ph.D. Thesis, Stanford University, SLAC-Report-320, 1987.

7. R.H. Schindler et ad., Phys. Rev. D24, 78 (1981).

8. M.E. Nelson et al., Phys. Rev. Lett. 50, 1542 (I983).

9. J.A. Jaros, Procedinge of the International Conference on Instrumentation for Colliding Beam Physice, edited by W. ARh, SI,AC-Report-250 (Stanford CA, 1982), p. 29.

10. See, for example: W. Burtel et al., Z. Phys. Cs3, 339 (1987).

11. L. Gladney et al., Phys. Rev. D34, 2601 (1986). 


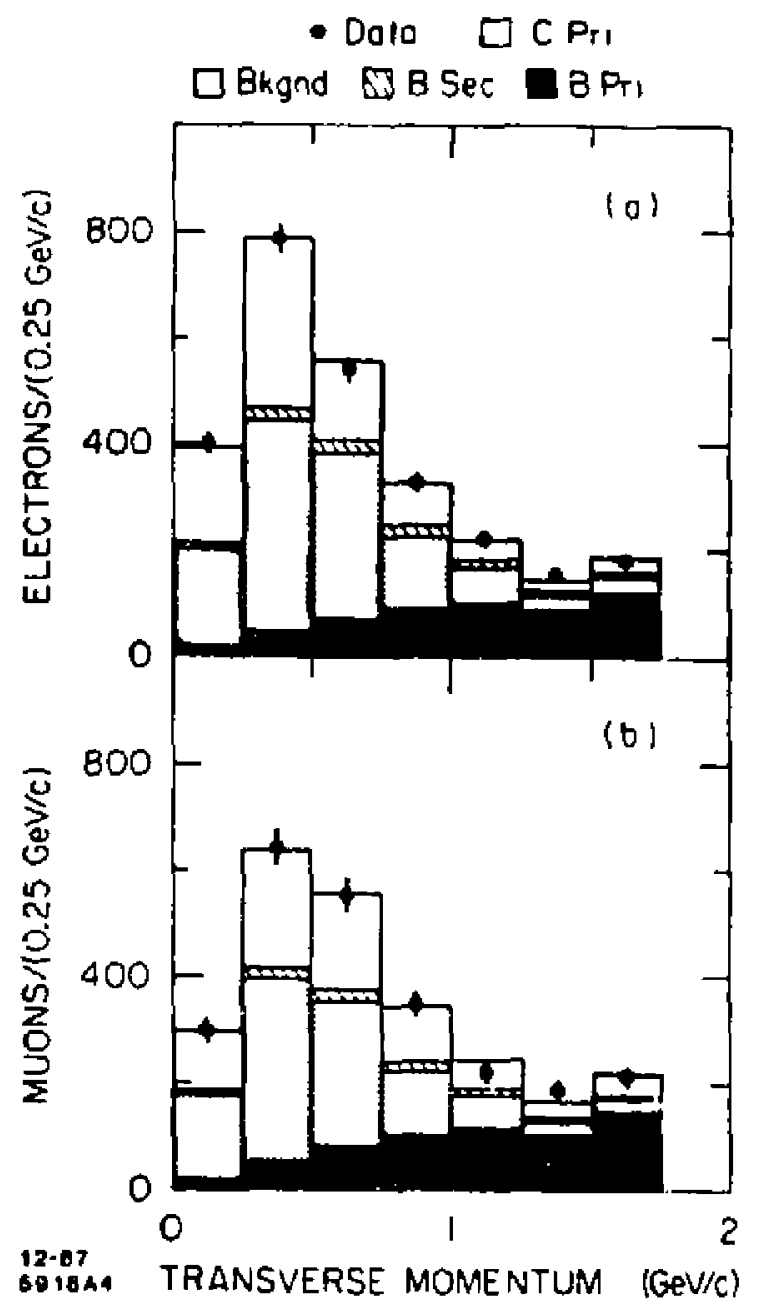

Figure 1: Results from the inclusive lepton analysis. Transveree momentum spectra for a) electrons and b) muons. The data is represented by points with error bara; the various components of the fit are represented by bargraphs. 


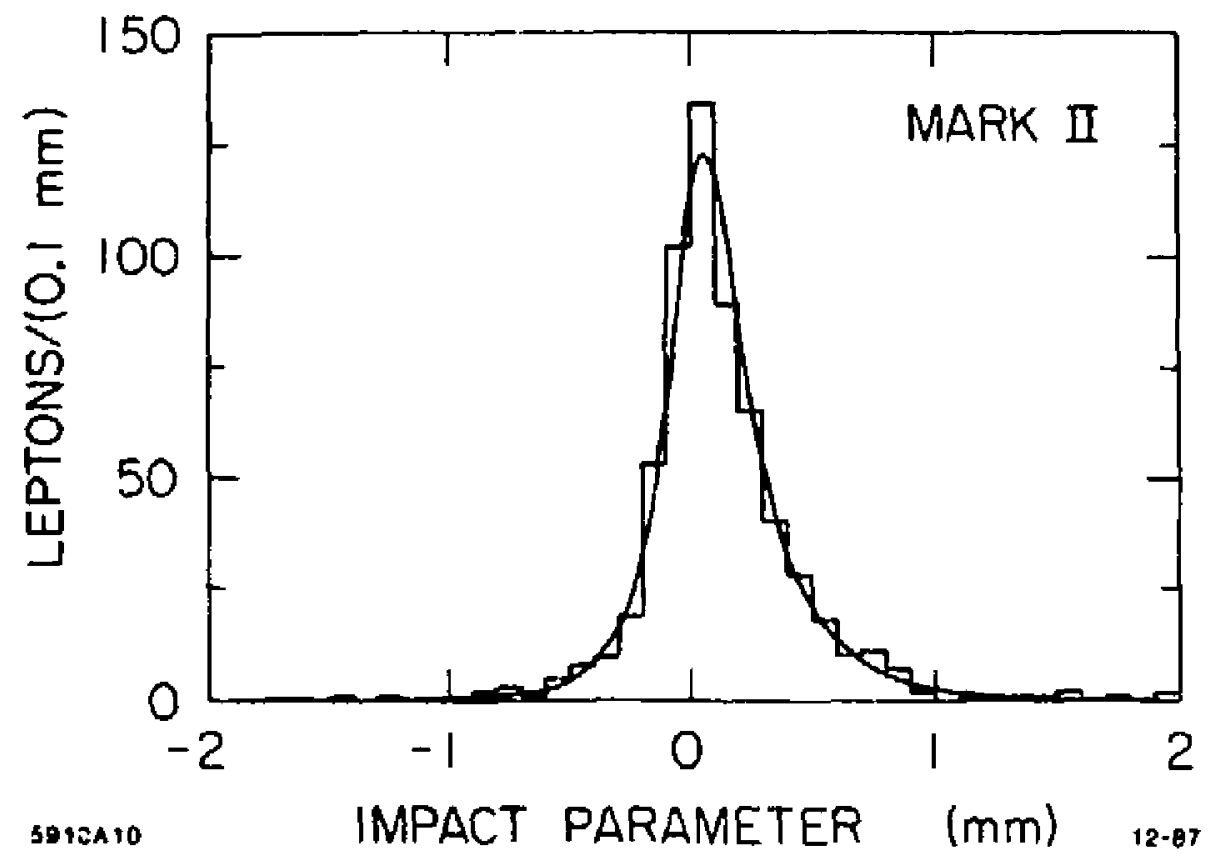

Figure e: lmpact parameter dintribution for the oample of high pt leptons. The curve corresponds to the maximum likelihood fit for the measured lifetime. 